\title{
Der Diskurs um Unterrichtsqualität in der Musikdidaktik zwischen generischen und fachspezifischen Dimensionen
}

\author{
Ulrike Kranefeld $\mathbb{D}$
}

Eingegangen: 23. Dezember 2020 / Überarbeitet: 9. März 2021 / Angenommen: 29. April 2021 / Online publiziert: 8. Juni 2021

(C) Der/die Autor(en) 2021

Zusammenfassung Der Artikel gibt Einblick in die Diskussion um Unterrichtsqualität in der Musikdidaktik. Dazu werden sowohl theoretische als auch empirische Beiträge gesichtet und begründet, warum aufgrund der Pluralität der Konzeptionen und Ansätze in der Musikdidaktik bislang kein verbindliches oder breit geteiltes Modell zur Unterrichtsqualität vorliegt. Am Beispiel der Zielperspektive einer „ästhetischen Musikpraxis“ wird gezeigt, wie theoretische Zugänge vor allem die Fachspezifik von Unterrichtsqualität herausarbeiten. Empirische Beiträge und hier besonders qualitative Videostudien stellen dagegen expliziten Bezug auch zu generischen Dimensionen von Unterrichtsqualität her, bemühen sich dabei aber um eine fachspezifische Modifikation. Vor dem Hintergrund des aktuellen musikdidaktischen Diskurses erfolgt abschließend ein Ausblick auf mögliche Kandidaten für weitere fachspezifische Ergänzungen und Ausdifferenzierungen für das Syntheseframework von Praetorius et al. (2020). Hier wird insbesondere die Ergänzung durch eine Dimension einer ,ästhetischen Aktivierung“ angeregt und auf die Berücksichtigung der materialen Dimension eines sinnvollen Umgangs mit Artefakten im Unterricht verwiesen.

Schlüsselwörter Musikdidaktik · Unterrichtsqualität · Unterrichtsforschung · Videographie $\cdot$ Ästhetische Aktivierung 


\title{
The music educational discourse on quality of teaching between generic and subject-specific dimensions
}

\begin{abstract}
This article delivers insight into the music educational discussion on quality of teaching. Therefore, a synopsis of theoretical and empirical publications is provided, offering a reason for the absence of a shared model for quality of teaching in music education due to the plurality of conceptions in this field. Using the example of 'aesthetic musical practice', it is shown that theoretical approaches underline the subject-specific nature of quality of teaching. Meanwhile, empirical publications, especially qualitative video studies, explicitly correspond to generic aspects of quality of teaching, albeit with an effort to adapt them to music education. Against the backdrop of this current music educational discourse, possible subjectspecific additions and differentiations of Praetorius' synthesis framework (2020) are presented and discussed. Special emphasis is put on the proposed dimension of 'aesthetic activation' and the consideration of material aspects of meaningful employment of artifacts in the music classroom.
\end{abstract}

Keywords Music education · Classroom research · Videographic approach · Teaching quality $\cdot$ Aesthetic acitvation

Bei der Sichtung der Diskussion zur Unterrichtsqualität innerhalb der Musikdidaktik ${ }^{1}$ stößt man - im Gegensatz zu einigen anderen Fachdidaktiken wie etwa Sport (Herrmann und Gerlach 2020) oder Geschichte (Zülsdorf-Kersting 2020) - weder auf theoretisch ausgearbeitete noch auf empirisch fundierte Modellierungen, die darauf ausgerichtet sind, fachbezogene Unterrichtsqualität übergreifend zu erfassen. Dieser Befund kann in einer Fachdidaktik verwundern, die innerhalb der nationalen Forschungslandschaft zur kulturellen Bildung nach einer Meta-Analyse von Liebau et al. (2014) gerade auch in Bezug auf empirische Zugänge als forschungsstark gilt und in der sich in den letzten Jahren eine differenzierte interview- und videobasierte Unterrichtsforschung entwickelt hat. Eine zentrale Ursache mag im vielfach konstatierten „Pluralismus“ (Jank 2020, S. 70) der Konzeptionen von Musikunterricht seit den 1960er-Jahren liegen, die in ihren unterschiedlichen Orientierungen und Zieldimensionen bis heute nebeneinanderstehen und damit auch eine verbindliche Bestimmung von Unterrichtsqualität oder zumindest einer ,,didaktischen Angemessenheit“ (Puffer und Hofmann 2016, S. 112) erschweren. Hinzu kommt ein stark divergierendes Gegenstandsverständnis, z. B. zwischen einer traditionellen Orientierung am Kunstwerk und der aktuell in der Musikdidaktik vielfach geteilten praxeologisch grundierten Vorstellung von Musik(en) als gesellschaftlich-kultureller Praxis (Jank et al. 2020).

\footnotetext{
1 Die Begriffe Musikdidaktik und Musikpädagogik werden uneinheitlich verwendet (Jank et al. 2020; vgl. auch Hörmann und Meidel 2016). Die folgenden Ausführungen zur Musikdidaktik beziehen sich in der Regel auf den wissenschaftlichen Zugriff auf allgemeinbildenden Musikunterricht, weniger auf außerschulische musikalische Bildung wie etwa den Instrumentalunterricht an Musikschulen, in der Unterrichtsqualität ebenso thematisiert wird (s. etwa Ernst 2007).
} 
Vor diesem Hintergrund überrascht es insgesamt nicht, dass die Musikdidaktik nicht ohne Weiteres ein konsensuales Modell zur Unterrichtsqualität vorlegen kann. Trotzdem zeigt der in (1) folgende Blick in den Diskurs um Unterrichtsqualität im Fach Musik, dass dieser zwar nicht systematisch geführt wird, allerdings in theoretischen Beiträgen insbesondere mit Blick auf die Fachspezifik mitverhandelt wird. Auch in empirischen Studien findet man - allerdings durchaus verstreut - Bezüge zu den in den Bildungswissenschaften diskutierten generischen Basisdimensionen von Unterrichtsqualität. Anschließend wird in (2) aus Perspektive der Autorin vor dem Hintergrund des aktuellen musikdidaktischen Diskurses ein Ausblick auf mögliche Kandidaten für weitere fachspezifische Ergänzungen und Ausdifferenzierungen für das Syntheseframework von Praetorius et al. (2020) gegeben, bevor ein abschließendes Fazit gezogen wird.

\section{Die Diskussion der Fachspezifität von Unterrichtsqualität in der Musik-Didaktik}

Im Folgenden soll an einem Beispiel aus dem aktuell in der Musikdidaktik vorherrschenden ästhetischen Paradigma exemplarisch gezeigt werden, wie in der theoretischen Diskussion die Frage nach ,gutem Musikunterricht“ im Sinne eines ,good teaching “ (Berliner 2005) $)^{2}$ implizit - mit starker Konzentration auf die Fachspezifik stets mitverhandelt wird. Anschließend werden bisherige Bezugnahmen der empirischen musikdidaktischen Forschung auf zentrale generische Basisdimensionen von Unterrichtsqualität wie „,kognitive Aktivierung“, ,Klassenführung“ und „konstruktive Unterstützung“ (Klieme et al. 2001) dargestellt.

\subsection{Das Mitverhandeln fachspezifischer Dimensionen von (Musik-)Unterrichtsqualität in theoretischen Überlegungen}

Mit Bezug auf die Geschichte der Musikdidaktik unterscheidet Kaiser (2005) vier Paradigmen für die Legitimation der Musik in der Allgemeinbildung: das Erziehungs- und Therapieparadigma, das anthropologische, das kulturtheoretische und das ästhetische Paradigma. Für die gegenwärtige Musikdidaktik konstatieren Jank et al. (2020) eine deutliche Tendenz zum ästhetischen Paradigma, das ,,musikalischästhetische Wahrnehmung und Erfahrung als einen besonderen, eigenständigen Modus der Welt- und Selbsterfahrung, der durch keinen anderen Modus ersetzt werden könne" (S. 267), beschreibt und ins Zentrum stellt. Es ist im Rahmen dieses Beitrags nicht möglich, die Pluralität der grundlagentheoretischen Überlegungen und ihres „Mitverhandelns“ von Unterrichtsqualität innerhalb der Musikdidaktik umfassend abzubilden. Deshalb soll im Folgenden das ästhetische Paradigma und die entsprechend dort vertretene Zieldimension, Qualitäten einer ästhetischen Musikpraxis ,im Unterricht erfahrbar werden zu lassen“ (Wallbaum 2005, S. 71), als Beispiel für die

\footnotetext{
2 Berliner (2005) unterscheidet zwei Perspektiven auf Unterrichtsqualität, das ,good teaching“, das die normativen Standards des jeweiligen Feldes erfüllt und ein „successful teaching“, das am Lernerfolg der Schüler*innen gemessen wird.
} 
Verhandlung von fachspezifischer Unterrichtsqualität in der Musikdidaktik dienen: Mit praxeologischem Gegenstandsverständnis fokussiert Rolle (2010) Prozessqualitäten, die mit der Prävalenz einer ,ästhetische[n] Musikpraxis“ (S. 246) im Unterricht verbunden sind. Dabei geht es ihm in seiner Konzeptualisierung von Musikunterricht als „Inszenierung ästhetischer Erfahrungsräume“ (Rolle 1999) darum, auf welche Art und Weise im Unterricht mit Musik umgegangen wird. Exemplarisch formuliert er deshalb für einzelne ,,Verhaltensweisen gegenüber Musik“ (Venus 1969)³ qualifizierende Merkmale:

Solange es beim Musikmachen nur darum geht, Töne in einem vorgegebenen Tempo und in der richtigen Reihenfolge vom Notenbild abzuspielen, handelt es sich noch nicht um ästhetische Musikpraxis; davon kann erst die Rede sein, wenn die Musizierenden sich bemühen, der Musik Ausdruck zu verleihen. (Rolle 2010, S. 246)

Ähnliche notwendige Bedingungen formuliert er für andere Kompetenzbereiche: So kommt es beim Musik-Erfinden nach Rolle erst dann zur ästhetischen Musikpraxis, wenn jenseits einer schematischen Erfüllung von Kompositionsregeln eigene „Gestaltungsspielräume“ (S. 247) genutzt werden. Beim Musikhören geht es nicht um eine bloße Analyse im Hinblick auf einzelne Elemente der Musik, sondern um die Möglichkeit, sich von der Musik ,sinnlich, emotional oder intellektuell, bewusst oder atmosphärisch (...) berühren zu lassen“ (ebd.). Und für den Bereich der Reflexion über Musik fordert er ,ästhetischen Streit“, mit „Begründungen, die unterschiedliche Sichtweisen und Interpretationen aufzeigen und vergleichen und für die eigenen werben“ (ebd.). Als Gegenbild nennt er die reine ,Vermittlung propositionalen Wissens über Musik“ (S. 246). Angesichts einer solchen Ausrichtung des Musikunterrichts auf ästhetische Musikpraxis schlagen Puffer und Hofmann (2017) vor, dass für den Musikunterricht die Basisdimension einer „,kognitiven Aktivierung“ in einem zukünftigen Modell zur fachbezogenen Unterrichtsqualität durch die einer „ästhetischen Aktivierung“ (S. 250) ergänzt werden sollte. Gleichzeitig deutet sich an, dass das Vorhandensein einer ästhetischen Aktivierung im Rahmen von Beobachtungen nicht durch eine einfache Kodierung musikbezogener Aktivität (etwa im Sinne vorhandener Musizierphasen) bestätigt werden kann, sondern dass Merkmale wie „Gestaltungsspielräume“ und ,ästhetischer Streit“" als hoch-inferente Kategorien einer theoretischen Ausdifferenzierung bedürfen, um etwa in einer quantifizierenden Unterrichtsqualitätsforschung genutzt werden zu können.

Die Merkmalsbestimmung einer ,ästhetischen Musikpraxis“ im Unterricht steht exemplarisch für eine Betonung der Fachspezifik in den theoretischen Überlegungen zur Unterrichtsqualität in der Musikdidaktik. Angesichts der oben beschriebenen Pluralität der musikdidaktischen Ansätze sei aber darauf verwiesen, dass andere Konzeptionen angesichts divergierender Zieldimensionen notwendigerweise zu anderen Relevanzsetzungen in Bezug auf Unterrichtsqualität kommen werden. So rückt

\footnotetext{
3 Einig ist man sich in der Musikdidaktik weitgehend über eine Fachsystematik, die sich an den ,,fünf vorrangigen Verhaltensweisen gegenüber Musik“ (Venus 1969) orientiert und mit der Produktion, Reproduktion, Rezeption, Transposition von Musik und Reflexion über Musik die Lehrpläne in den Bundesländern (teilweise in leicht veränderter Form) bis heute prägt.
} 
in einer Konzeption wie dem „Aufbauenden Musikunterricht“ (Jank 2020), der auf das Musik-Lernen im Sinne eines progressiven Erwerbs musikalischer Fähigkeiten setzt, z.B. eine Dimension wie das Üben im Musikunterricht mehr in den Fokus der Betrachtung als bei der oben dargestellten „Inszenierung ästhetischer Erfahrungsräume“. Systematisch werden die Qualitätsdimensionen von Musikunterricht aber in keiner der vorliegenden musikdidaktischen Konzeption soweit aufeinander bezogen, dass bereits von einem Modell von Unterrichtsqualität gesprochen werden kann.

Auch wenn aufgrund der variierenden Zieldimensionen kein konsensuales Modell zur Unterrichtsqualität im Fach Musik vorliegt, besteht aktuell Einigkeit in der Forderung nach einem vielfältigen Umgang mit Musik und der besonderen Bedeutung der Fachpraxis im Sinne von Musizieren, Komponieren, Improvisieren und Singen. Krupp (2021) verweist in ihrem summarischen Blick auf Qualitätsmerkmale von Musikunterricht zudem auf weitere ,zentrale Linien“ (S. 171), die innerhalb der aktuellen Diskussion in der Musikpädagogik geteilt werden und eine fachspezifische Ergänzung gängiger bildungswissenschaftlicher Modelle zur Unterrichtsqualität anregen können. So nennt sie etwa im Hinblick auf die Auswahl von Inhalten die „musikalische Vielfalt als Normalfall“ (S. 172) und betont in Anschluss an Stöger (2021) die besondere Bedeutung eines sensiblen Umgangs mit (Be-)Wertungen, Zuschreibungen und Stereotypenbildungen im Fach Musik.

\subsection{Zur musikdidaktischen Adaption generischer Basisdimensionen in empirischen Arbeiten}

Anders als in theoretischen Beiträgen finden sich in empirisch ausgerichteter musikdidaktischer Forschung durchaus explizite Bezugnahmen zu generischen Dimensionen von Unterrichtsqualität, auch wenn es sich dabei eher um verstreute (Be-)Funde handelt und weniger um einen expliziten Forschungsstrang zur Unterrichtsqualität. Methodischen Zugang zu den Basisdimensionen der „,kognitiven Aktivierung“, „konstruktiven Unterstützung“ und „Klassenführung“ (Klieme et al. 2001) nimmt die Forschung dabei einerseits durch die Befragung der Lehrpersonen und Schüler*innen, andererseits durch qualitative Interview- und Videostudien: Interessanterweise erscheinen diese fast ausschließlich in einem relativ spezifischen Bereich der musikdidaktischen Unterrichtsforschung, der sich für die Frage nach dem Status und der Rolle von musikalischer Fachpraxis im Unterricht (im Sinne von Musizieren, Komponieren, Improvisieren und Singen) interessiert und weniger Phasen der expliziten Rezeption von und Reflexion über Musik im Blick hat. Ein Grund dafür mag darin liegen, dass seit einigen Jahren durch Kooperationsmodelle mit außerschulischen Partnern wie z. B. Musikschulen institutionell verankerte Formate eines sog. „erweiterten Musikunterrichts“ in den Schulen Einzug halten, die das Erlernen eines Musikinstruments oder das Singen ins Zentrum stellen. Das betrifft für die Grundschule zum Beispiel Programme wie Jedem Kind ein Instrument (JeKi) und in der Sekundarstufe vielfältige Formate von sogenannten Musikklassen. Der vom BMBF von 2009-2016 geförderte Forschungsschwerpunkt zu JeKi trug zur entsprechenden thematischen Ausrichtung der Forschung bei. Diskussionen um Unterrichtsqualität entzünden sich nun angesichts einer manchmal einseitigen Fokussierung auf den Bereich der Produktion nicht mehr nur an der Frage, was ein guter, sondern auch 
daran, was unter diesen Bedingungen ein allgemeinbildender Musikunterricht sein kann (Geuen 2012; Wallbaum 2005; Kaiser 2010).

Die empirischen Beiträge aus der Musikdidaktik unterscheiden sich bei ihren Bezugnahmen auf die generischen Basisdimensionen im Grad ihrer fachspezifischen Adaption: Nur vereinzelt findet man Beiträge, die die drei Basisdimensionen ohne weitere fachdidaktische Anpassung nutzen und bei Erhebungen unmittelbar auf die in den Bildungswissenschaften etablierten Instrumente zurückgreifen, so etwa in einer quantitativen Befragung von Haas et al. (2019), die die jeweilige Unterrichtsqualität in Regel- und Musikklassen aus Schülersicht vergleichen ${ }^{4}$ oder bei Heß (2018), die - ebenfalls aus Schülersicht - die „pädagogische Unterrichtsqualität“ (S. 76) besonders im Hinblick auf das Unterrichtsklima in ihrem Einfluss auf die Facheinstellungen bei den Schüler*innen misst ${ }^{5}$. Auch Niessen $(2010,2011)$ nutzt explizit die „Grunddimensionen der Unterrichtsqualität“ nach Klieme und Rakoczy (2008, S. 224) als Folie für ihre qualitative Auswertung von Interviewmaterial mit einer Musiklehrerin und den Schüler*innen und verfolgt dabei vor allem die Frage nach kognitiver Aktivierung und Verarbeitungstiefe.

Dagegen machen qualitative Studien, die videobasiert arbeiten und die sich dabei explizit auf die drei Basisdimensionen von Unterrichtsqualität richten, vor allem deren jeweilige fachspezifische Modifizierung und Ausdifferenzierung zum Gegenstand ihrer Überlegungen, wie folgende Beispiele zeigen: So spricht etwa Gebauer (2013) in ihrer videobasierten Studie davon, ,kognitive Aktivierung fachspezifisch zu konkretisieren“ (S. 63) und geht der Frage nach, inwieweit die jeweilige Einbettung musikpraktischer Phasen in die Stundenverläufe auf eine Verarbeitungstiefe in der Auseinandersetzung mit dem Unterrichtsinhalt abzielen. Bezogen auf die oben genannte Fachsystematik betrifft dies die zentrale fachdidaktische Frage nach einer sinnvollen Verbindung von Produktion und Reflexion von Musik im Unterricht. Eine Studie von Kranefeld und Mause (2020) nimmt mit der Erforschung der Lernbegleitung in Gruppenkompositionsprozessen einen Teilaspekt der Dimension „Konstruktive Unterstützung“ als Qualitätsdimension in den Blick und rekonstruiert videobasiert Praktiken von Musiklehrenden und Komponist*innen im fachspezifisch besonders akzentuierten Spannungsfeld der Lernbegleitung zwischen Anleitung und Eigen-Sinn. Zudem richten sich einige Studien auf die besonderen fachspezifischen Bedingungen von Differenzkonstruktion im Musikunterricht, etwa angesichts differierender musikalischer Vorerfahrungen (Heberle 2019; Kranefeld und Heberle 2020). Auch für die Dimension „Klassenführung“ liegen aus dem BMBF-Forschungsschwerpunkt zu Jedem Kind ein Instrument (JeKi) videobasierte Befunde zur fachspezifischen Steuerung von Übergängen und zur Aufrechterhaltung des Gruppen-Fokus vor (Kranefeld et al. 2015a, 2015b). Hierfür wurden Analysedimensionen in Anlehnung an Thiel et al. (2012) und Kounin (2006) aufgegriffen und angesichts der besonderen (auch akustischen) Bedingungen eines Musizierens in Gruppen fachspezifisch gewendet. Zudem rückt aktuell die materiale Dimension des Musikunterrichts in den Fokus videographischer Studien mit der Frage, wie an

\footnotetext{
4 Sie orientieren sich dabei an Baumert et al. (2009) und Fauth et al. (2014).

5 Sie nutzt zur Erfassung des Schülerbefindens Items aus Rakoczy et al. (2008) und für die pädagogische Kompetenz der Lehrperson aus Wagner et al. (2009).
} 
und mit Artefakten gelernt werden kann, also etwa in Bezug auf digitale Medien (Duve 2020), Musikinstrumente oder Alltagsgegenstände (Kranefeld et al. 2019; Langner 2020).

Der Mehrwert dieser qualitativen Videostudien liegt im hohen Auflösungsgrad ihrer mikroanalytischen Perspektive, die Unterrichtsqualität - im ursprünglichen Sinne von qualitas als Beschaffenheit - in einzelnen Dimensionen fachspezifisch auszudifferenzieren vermag.

$\mathrm{Zu}$ vermuten ist, dass die in den letzten Jahren verstärkte Bezugnahme empirischer Studien auf generische Qualitätsdimensionen und damit einer evaluativen Perspektive sowohl mit der Anforderung korrespondiert, empirisch fundierte Kompetenzmodelle und Leistungstests für Schüler*innen (Jordan et al. 2012; Hasselhorn und Lehmann 2015) und Musiklehrkräfte (Puffer und Hofmann 2016, 2017) zu entwickeln als auch mit dem Anspruch, bei der systematischen Beforschung des Musikunterrichts jeweils Anschluss an den aktuellen Stand der empirischen Forschung in den Bildungswissenschaften zu nehmen.

\section{Eigene Positionierung zur Frage der Fachspezifität von Unterrichtsqualität}

Aufgrund des Fehlens eines ausgearbeiteten und geteilten Modells zur Unterrichtsqualität im Musikunterricht kann die aktuell von Praetorius et al. (2020) auf der Basis des Syntheseframeworks (Praetorius und Charalambous 2018) erarbeitete Synopse für fachdidaktische Unterrichtsqualität für die Naturwissenschaften, Sport und Geschichte für die Musik nicht systematisch fortgeschrieben werden. Gleichwohl sollen abschließend vor dem Hintergrund des oben dargestellten aktuellen musikdidaktischen Diskurses und der dort thematisierten Dimensionen von Unterrichtsqualität schlaglichtartig und exemplarisch einige ausgewählte Kandidaten für fachspezifische Ausdifferenzierungen des Syntheseframeworks diskutiert werden. ${ }^{6}$ Gegenüber den drei Basisdimensionen modelliert das Syntheseframework sieben Dimensionen: (I) Auswahl und Thematisierung von Inhalten und Fachmethoden, (II) kognitive Aktivierung, (III) Unterstützung des Übens, (IV) formatives Assessment, (V) Unterstützung des Lernens aller Schülerinnen und Schüler, (VI) sozio-emotionale Unterstützung und (VII) Klassenführung (Praetorius et al. 2020). Die fachspezifischen Ausdifferenzierungen angesichts des oben dargestellten aktuellen musikdidaktischen Diskurses finden sich - ähnlich wie in den bisherigen Befunden erwartet (Praetorius et al. 2020) - hauptsächlich auf der Ebene von fachspezifischen Indikatoren. Zudem können einige fachspezifische Ergänzungen der anderen Fächer geteilt werden. In zwei Aspekten, der ästhetischen Aktivierung und der materialen Dimension des Unterrichts, werden zwei Ergänzungen vorgeschlagen:

- Konsens besteht in der Musikdidaktik in Bezug auf die Inhalte (Dimension I) in der Forderung nach einer „musikalische[n] Vielfalt als Normalfall“ (Krupp 2021,

\footnotetext{
${ }^{6}$ Für konstruktive Diskussionen danke ich den Kolleg*innen aus der Musikpädagogik Thade Buchborn, Bernhard Hofmann, Valerie Krupp, Gabriele Puffer und Malte Sachsse.
} 
S. 172), die ggf. als fachspezifische Ergänzung bzw. als Indikator für eine „Auswahl von bedeutungsvollen, dem Lernstand angemessenen Inhalten sowie Fachmethoden“ (Dimension I. 1, Praetorius et al. 2020, S. 417) für die Musik betrachtet werden kann. Eine solche Dimension der Unterrichtsqualität wäre allerdings nicht auf der Ebene der Einzelstunde, sondern nur über einen längeren Beobachtungszeitraum bestimmbar.

- Aus dem oben angedeuteten und noch weitgehend unausgearbeiteten Konstrukt der ,ästhetischen Aktivierung“ (Puffer und Hofmann 2017) ließe sich zumindest ein Kandidat für eine zentrale Ergänzung des bestehenden Syntheseframeworks für die Musik ableiten bzw. konstatieren, dass eine wesentliche Qualitätsdimension der ästhetischen Fächer dort bislang nicht abgebildet wird. Auch wenn auf den ersten Blick eine begriffliche Nähe zur Dimension II Kognitive Aktivierung besteht, ist fraglich, ob eine ästhetische Aktivierung aufgrund der angenommenen prinzipiellen Varianz ,ästhetischer Rationalität“ (Rolle 2010) hier untergeordnet werden kann oder nicht vielmehr nebengeordnet werden müsste. Noch liegt ein Konstrukt wie die ästhetische Aktivierung quer zur stark an den MINT-Fächern orientierten Logik einer „kognitiven Rationalität“, die nach Baumert et al. (2001, S. 21) schon im Kontext von PISA den Blick auf Unterricht dominiert und andere „Modi der Welterfahrung“, etwa eine ,„ästhetisch-expressive (...) Rationalität“ (ebd.) nicht in gleichem Maße berücksichtigt (s. dazu auch Rolle 2010; Wallbaum 2010). Denkbar ist auch, dass nach einer genaueren, noch ausstehenden theoretischen Ausarbeitung des Konstrukts deutlich wird, dass einzelne Subdimensionen einer ästhetischen Aktivierung wiederum unterschiedlichen bestehenden Dimensionen des Syntheseframeworks zugeordnet werden können, auch wenn dann zu prüfen wäre, ob eine jeweilige „Konstruktäquivalenz“ (Praetorius et al. 2020, S. 427) dabei tatsächlich noch besteht.

- Anschluss nehmen kann die Musikdidaktik am Vorschlag aus dem Sport (Herrmann und Gerlach 2020), das Konstrukt der Kognitiven Aktivierung (Dimension II) angesichts instrumentalen und vokalen Lernens und des Bereichs „Musik und Bewegung“ um eine für die Musik senso-motorische Komponente zu erweitern. Entsprechend wird gerade auch in musikalischen Praxisphasen die Dimension III Unterstützung des Übens ähnlich wie im Sport in besonderer Weise relevant und zudem hinsichtlich der Fehlerkultur reflektiert (Spychiger 2014).

- Ähnlich wie im Sport werden auch in der Musik Aspekte der sozio-emotionalen Unterstützung (Dimension VI) als fachdidaktisch besonders bedeutsam für Unterrichtsqualität eingeschätzt (Krupp 2021, S. 172). Das betrifft etwa eine besondere Sensibilität, die Lehrende wie Schüler*innen sich gegenseitig angesichts ,der hohen Bedeutung von Wertungen und Bewertungen im Feld der Musik“ und angesichts der „Diversität an Vorerfahrungen und Interessen“ (Stöger 2021, S. 141) entgegenbringen müssen. Das gilt besonders für Situationen, in denen der für eine ästhetische Musikpraxis geforderte ,ästhetische Streit“" zentral wird. Ebenso wie im Sport wird außerdem die körperliche - und im Falle der Musik auch akustische „Exponiertheit“ (Herrmann und Gerlach 2020, S. 378) und die damit verbundene Gefahr der Beschämung zu einer besonderen Herausforderung in musikalischen Praxisphasen, wie die videographischen Studien zur Differenzkonstruktion zeigen (Heberle 2019; Kranefeld und Heberle 2020). 
- Der von den Naturwissenschaften und dem Sport in der Dimension VII Klassenführung ergänzte Aspekt „Raum- und Materialmanagement“ (Praetorius et al. 2020, S. 421) ist auch für den Musikunterricht bedeutsam, bildet aber womöglich nur Teilaspekte der Herausforderung ab, die die Organisation und Durchführung von Musizierphasen im Unterricht darstellen. So konnte Kranefeld (2016) die Dimension des „Beschäftigungsradius“ (Kounin 2006, S. 120)7 im Spannungsfeld von Einzel- und Gruppenbetreuung beim instrumentalen Gruppenunterricht fachspezifisch ausdifferenzieren. Puffer und Hofmann (2017) diskutieren bei ihrer Modellierung des „,domänenspezifischen Professionswissens von Musiklehrkräften“ (S. 254) sogar, ob Aspekte der Klassenführung bei Musiklehrer*innen dem pädagogischen Wissen zuzuordnen sind oder doch eher - aufgrund der besonderen Anforderungsstruktur beim instrumentalen Klassenmusizieren - als neue Facette innerhalb des fachdidaktischen Wissens modelliert werden müssten. Eine Bezeichnung „Klassenführung in Musik“ würde dieser Überlegung auch im Syntheseframework Rechnung tragen und zugleich dem Vorschlag von Praetorius et al. (2020) folgen, eine Fachspezifik zu markieren, wenn es sich nach einer fachspezifischen Konkretisierung nicht mehr um das gleiche Konstrukt handelt (S. 427).

- In diesem Kontext stellt sich auch die meist nur fachdidaktisch und angesichts des jeweiligen Lerngegenstands zu beantwortende Frage nach der materialen Dimension des Unterrichts, die - wie oben gezeigt - aktuell vermehrt Gegenstand musikdidaktischer Forschung wird. Während Praetorius et al. (2020) im Anschluss an Reusser (2008) von der Vorstellung eines didaktischen Dreiecks ausgehen, erscheint es für die Musikdidaktik lohnenswert im ,didaktischen Tetraeder“ (Hußmann et al. 2018) zu denken, der neben Lehrenden, Schüler*innen und dem Lerngegenstand auch die Artefakte berücksichtigt und dies nicht nur in der Dimension eines bloßen „Raum- und Materialmanagements“, sondern bezogen auf ein Lernen mit und an den Dingen, auch im (Musik-)Unterricht (Kranefeld et al. 2019; Langner 2020). Eine (Sub-)Dimension zum sinnvollen Einsatz von Artefakten könnte auch fachübergreifend als Ergänzung sowohl in Dimension I Auswahl und Thematisierung von Inhalten und Fachmethoden, II Kognitive Aktivierung als auch in der Dimension V Unterstützung des Lernens aller Schüler*innen relevant sein.

- Für das Fach Geschichte wurde eine das Syntheseframework ergänzende Dimension der „Diskursqualität“ als ein „,quer liegendes Merkmal“ (Zülsdorf-Kersting 2020, S. 403) angeregt, das auch in einem zentralen fachdidaktischen Konstrukt wie dem ,ästhetischen Streit“ (Rolle 2010) im Musikunterricht aufscheint. Darüber hinaus ist für die Musik aber auch eine non-verbale Kommunikationsqualität zu berücksichtigen und ggf. zu ergänzen, die in der musikdidaktischen Forschung aktuell unter ,interpersoneller Koordination“ (Hellberg 2019) im Ensemblespiel beforscht wird und eine mit kaum einem anderen Fach (außer vielleicht dem Sport) vergleichbare ,präzise und flexible Abstimmung der Aktivitäten“ (S. 20) der beteiligten Akteur*innen erfordert.

\footnotetext{
7 Unter „Beschäftigungsradius“ versteht Kounin (2006, S. 120) die „Arbeitsanforderungen (...), die dann, wenn ein Schüler aufgerufen wird, an die übrigens Kinder gestellt werden“.
} 
Es ließen sich sicherlich weitere fachspezifische Ergänzungen diskutieren. Insbesondere erscheint die weitere fachspezifische Konkretisierung bzw. Ausdifferenzierung auf Indikatorenebene notwendig, die oben für die Dimensionen IV und V nur angedeutet werden konnte. Offen bleibt die Frage nach der Sensibilität des Syntheseframeworks für die unterschiedlichen Bereiche der Fachsystematik Musik zwischen Produktion, Rezeption und Reflexion. So wäre etwa die genannte Erweiterung der kognitiven Aktivierung durch eine senso-motorische Aktivierung insbesondere für fachpraktische Phasen des Musizierens und des Bewegens zur Musik besonders angemessen, nicht aber als allgemeines Merkmal von Unterrichtsqualität im $\mathrm{Mu}-$ sikunterricht anzusehen. Schon Praetorius et al. (2020) weisen darauf hin, dass sich Fachdidaktiken in ihren Forschungen zur Unterrichtsqualität nicht von den bildungswissenschaftlich ausgearbeiteten Basisdimensionen, sondern eher von ihrer eigenen Fachsystematik leiten lassen (S. 431).

\section{Fazit und Ausblick}

Da ein umfassendes, konsensuales Modell von Unterrichtsqualität und seine empirische Beforschung aufgrund der Pluralität der Ansätze in der Musikdidaktik in naher Zukunft wohl nicht zu erwarten ist, kommt sowohl der qualitativen Unterrichtsforschung als auch theoretischen Arbeiten die wichtige Aufgabe zu, generische wie auch einzelne fachspezifische Dimensionen von Unterrichtsqualität weiter auszudifferenzieren. Ein begleitender Schritt könnte - ggf. auch gemeinsam mit anderen ästhetischen Fächern - in der Konkretisierung, theoretischen Ausarbeitung und Operationalisierung einer Dimension ästhetischer Aktivierung bestehen. Ein Mitbedenken ästhetischer Aspekte könnte durchaus auch wiederum die Perspektive vermeintlich „entfernterer“ Fächer befruchten, wenn man etwa in der Mathematikdidaktik an eine mögliche „soziomathematische Norm“ denkt, die eine „elegante Lösung“ einer bloß „richtigen“" vorziehen könnte (Erath 2017, S. 31).

Funding Open Access funding enabled and organized by Projekt DEAL.

Open Access Dieser Artikel wird unter der Creative Commons Namensnennung 4.0 International Lizenz veröffentlicht, welche die Nutzung, Vervielfältigung, Bearbeitung, Verbreitung und Wiedergabe in jeglichem Medium und Format erlaubt, sofern Sie den/die ursprünglichen Autor(en) und die Quelle ordnungsgemäß nennen, einen Link zur Creative Commons Lizenz beifügen und angeben, ob Änderungen vorgenommen wurden.

Die in diesem Artikel enthaltenen Bilder und sonstiges Drittmaterial unterliegen ebenfalls der genannten Creative Commons Lizenz, sofern sich aus der Abbildungslegende nichts anderes ergibt. Sofern das betreffende Material nicht unter der genannten Creative Commons Lizenz steht und die betreffende Handlung nicht nach gesetzlichen Vorschriften erlaubt ist, ist für die oben aufgeführten Weiterverwendungen des Materials die Einwilligung des jeweiligen Rechteinhabers einzuholen.

Weitere Details zur Lizenz entnehmen Sie bitte der Lizenzinformation auf http://creativecommons.org/ licenses/by/4.0/deed.de. 


\section{Literatur}

Baumert, J., Stanat, P., \& Demmrich, A. (2001). PISA 2000: Untersuchungsgegenstand, theoretische Grundlagen und Durchführung der Studie. In PISA-Konsortium (Hrsg.), PISA 2000. Basiskompetenzen von Schülerinnen und Schülern im internationalen Vergleich. Opladen: Leske + Budrich.

Baumert, J., Blum, W., Brunner, M., Dubberke, T., Jordan, A., Klusmann, U., Krauss, S., Kunter, M., Löwen, K., Neubrand, M., \& Tsai, Y. M. (2009). Professionswissen von Lehrkräften, kognitiv aktivierender Mathematikunterricht und die Entwicklung von mathematischer Kompetenz (COACTIV). Dokumentation der Erhebungsinstrumente. Berlin: Max-Planck-Institut für Bildungsforschung.

Berliner, D.C. (2005). The near impossibility of testing for teacher quality. Journal of Teacher Education, $56,205-213$.

Duve, J. (2020). Komponieren am Raster. Fallanalytische Perspektiven auf Prozesse des Musik-Erfindens mit digitalen Medien. In U. Kranefeld \& J. Voit (Hrsg.), Musikunterricht im Modus des Musik-Erfindens. Fallanalytische Perspektiven. Münster: Waxmann.

Erath, K. (2017). Mathematisch diskursive Praktiken des Erklärens. Rekonstruktion von Unterrichtsgesprächen in unterschiedlichen Mikrokulturen. Wiesbaden: Springer.

Ernst, A. (2007). Was ist guter Instrumentalunterricht? Beispiele und Anregungen. Aarau: Nepomuk.

Fauth, B., Decristan, J., Riesner, S., Klieme, E., \& Büttner, G. (2014). Student ratings of teaching quality in primary school: dimensions and predictions of student outcomes. Learning and Instruction, 29, $1-9$.

Gebauer, H. (2013). „Beschreibt doch mal die Form, die wir gerade gemacht haben.“ Kognitive Aktivierung im Musikunterricht. In A. Lehmann-Wermser \& M. Krause-Benz (Hrsg.), Musiklehrer(-bildung) im Fokus musikpädagogischer Forschung (S. 61-79). Münster: Waxmann.

Geuen, H. (2012). Klassenmusizieren oder allgemeinbildender Musikunterricht? Plädoyer für einen vielfältigen schulischen Musikunterricht. In M. Pabst-Krüger \& J. Terhag (Hrsg.), Musikunterricht heute 9. Musizieren mit Schulklassen. Praxis - Konzepte - Perspektiven (S. 48-63). Berlin: Lugert.

Haas, M., Nonte, S., Krieg, M., \& Stubbe, T.C. (2019). Unterrichtsqualität in Musikklassen. Befunde aus der quasi-experimentellen Studie ProBiNi. In V. Weidner \& C. Rolle (Hrsg.), Praxen und Diskurse aus Sicht musikpädagogischer Forschung (S. 137-154). Münster: Waxmann.

Hasselhorn, J., \& Lehmann, A. (2015). Leistungsheterogenität im Musikunterricht. Eine empirische Untersuchung zu Leistungsunterschieden im Bereich der Musikpraxis in Jahrgangsstufe 9. In A. Niessen \& J. Knigge (Hrsg.), Theoretische Rahmung und Theoriebildung in der musikpädagogischen Forschung (S. 163-176). Münster: Waxmann.

Heberle, K. (2019). Zur Konstruktion von Leistungsdifferenz im Rahmen musikpädagogischer Unterrichtspraxis. Eine Videostudie zum instrumentalen Gruppenunterricht in der Grundschule. Münster: Waxmann.

Hellberg, B. (2019). Koordinationsprozesse beim Musizieren im Instrumentalen Gruppenunterricht. Münster: Waxmann.

Herrmann, C., \& Gerlach, E. (2020). Unterrichtsqualität im Fach Sport. Ein Überblicksbeitrag zum Forschungsstand in Theorie und Empirie. Unterrichtswissenschaft, 48, 361-384.

Heß, F. (2018). Gendersensibler Musikunterricht. Empirische Studien und didaktische Konsequenzen. Wiesbaden: Springer.

Hörmann, S., \& Meidel, E. (2016). Orientierung im Begriffsdschungel - terminologische und fachstrukturelle Perspektiven zur Profilierung der Musikpädagogik und Musikdidaktik. In B. Clausen, A. Cvetko, S. Hörmann, M. Krause-Benz \& S. Kruse-Weber (Hrsg.), Grundlagentexte Wissenschaftlicher Musikpädagogik. Begriffe, Positionen, Perspektiven im systematischen Fokus (S. 11-68). Münster: Waxmann.

Hußmann, S., Kranefeld, U., Kuhl, J., \& Schlebrowski, D. (2018). Das geschachtelte Tetraeder und inklusionsorientierte Designprinzipien als Modell für Entwicklung und Forschung in einer inklusionsorientierten Lehrerinnen- und Lehrerbildung. In S. Hußmann \& B. Welzel (Hrsg.), DoProfiL - Das Dortmunder Profil für inklusionsorientierte Lehrerinnen- und Lehrerbildung (S. 11-26). Münster: Waxmann.

Jank, W. (Hrsg.). (2020). Musik-Didaktik. Praxishandbuch für die Sekundarstufe I und II (8. Aufl.). Berlin: Cornelsen.

Jank, W., Knigge, J., \& Niessen, A. (2020). Musikdidaktik. Bestandsaufnahme und Forschungsperspektiven. In M. Rothgangel, U. Abraham, H. Bayrhuber, V. Frederking, W. Jank \& H. J. Vollmer (Hrsg.), Lernen im Fach und über das Fach hinaus. Bestandsaufnahme und Forschungsperspektiven aus 17 Fachdidaktiken im Vergleich (S. 262-288). Münster: Waxmann. 
Jordan, A.-K., Knigge, J., Lehmann, A.C., Niessen, A., \& Lehmann-Wermser, A. (2012). Entwicklung und Validierung eines Kompetenzmodells im Fach Musik - Wahrnehmen und Kontextualisieren von Musik. Zeitschrift für Pädagogik, 58(4), 500-521.

Kaiser, H.J. (2005). Musikerziehung/Musikpädagogik. In S. Helms, R. Schneider \& R. Weber (Hrsg.), Lexikon der Musikpädagogik (S. 166-169). Kassel: Bosse.

Kaiser, H. J. (2010). Verständige Musikpraxis. Eine Antwort auf Legitimationsdefizite des Klassenmusizierens. Zeitschrift für Kritische Musikpädagogik, 47-68. https://www.zfkm.org/10-kaiser.pdf.

Klieme, E., \& Rakoczy, K. (2008). Empirische Unterrichtsforschung und Fachdidaktik. Outcome-orientierte Messung und Prozessqualität des Unterrichts. Zeitschrift für Pädagogik, 54(2), 222-237.

Klieme, E., Schümer, G., \& Knoll, S. (2001). Mathematikunterricht in der Sekundarstufe I. „Aufgabenkultur" und Unterrichtsgestaltung. In E. Klieme \& J. Baumert (Hrsg.), TIMSS-Impulse für Schule und Unterricht. Forschunsbefunde, Refominitiativen, Praxisberichte und Video-Dokumente (S. 43-57). Bonn: Bundesministerium für Bildung und Forschung.

Kounin, J.S. (2006). Techniken der Klassenführung. Standardwerke aus Psychologie und Pädagogik Reprints, Bd. 3. Münster: Waxmann.

Kranefeld, U. (2016). Herzstück Musizieren? Ein empirischer Blick auf Handlungs- und Orientierungsmuster von Lehrenden im instrumentalen Gruppenunterricht. In B. Wüstehube, Ch Stöger, P. Röbke \& N. Ardila-Mantilla (Hrsg.), Herzstück Musizieren. Instrumentaler Gruppenunterricht zwischen Planung und Wagnis. Texte zur Instrumentalpädagogik (S. 13-31). Mainz: Schott.

Kranefeld, U., \& Heberle, K. (2020). Passungsprozesse im Musikunterricht. Videobasierte Fallanalysen zur Differenzbearbeitung in musikpädagogischen Angeboten der 5. und 6. Klasse. Münster: Waxmann.

Kranefeld, U., \& Mause, A.-L. (2020). Anleitung zum Eigen-Sinn? Ergebnisse einer videobasierten Studie zur Begleitung von Gruppenprozessen des Musik-Erfindens. In S. Timm, J. Costa, C. Kühn \& A. Scheunpflug (Hrsg.), Kulturelle Bildung. Theoretische Perspektiven, methodologische Herausforderungen, empirische Befunde (S. 113-129). Münster: Waxmann.

Kranefeld, U., Busch, T., \& Dücker, J. (2015a). Instrumentaler Gruppenunterricht in der Grundschule. Teilnahme, Selbstkonzepte, Gestaltungsmuster. In U. Kranefeld (Hrsg.), Instrumentalunterricht in der Grundschule. Prozess- und Wirkungsanalysen zum Programm Jedem Kind ein Instrument. Bildungsforschung; 41. (S. 49-89). Berlin: BMBF.

Kranefeld, U., Heberle, K., \& Naacke, S. (2015b). Videographische Befunde zu Aspekten von Unterrichtsqualität im ersten JeKi-Jahr. In U. Kranefeld (Hrsg.), Instrumentalunterricht in der Grundschule. Prozess- und Wirkungsanalysen zum Programm Jedem Kind ein Instrument. Empirische Bildungsforschung. (S. 147-165). Berlin: BMBF.

Kranefeld, U., Mause, A.-L., \& Duve, J. (2019). Zur Materialität von Prozessen des Musik-Erfindens. Interaktionsanalytische Zugänge zur Wandelbarkeit der Dinge. In V. Weidner \& C. Rolle (Hrsg.), Praxen und Diskurse aus Sicht musikpädagogischer Forschung (S. 35-50). Münster: Waxmann.

Krupp, V. (2021). Perspektiven auf Wirksamkeit - ein Resümee. In V. Krupp (Hrsg.), Wirksamer Musikunterricht (S. 168-175). Hohengehren: Schneider.

Langner, J. (2020). „Wenn ihr da 'n Keyboard wollt“. Bedeutungszuschreibungen bei der Auswahl von Instrumenten im Kontext des Musik-Erfindens. In U. Kranefeld \& J. Voit (Hrsg.), Musikunterricht im Modus des Musik-Erfindens. Fallanalytische Perspektiven (S. 81-96). Münster: Waxmann.

Liebau, E., Jörissen, B., \& Klepacki, L. (Hrsg.). (2014). Forschung zur kulturellen Bildung. Grundlagenreflexionen und empirische Befunde. München: kopaed.

Niessen, A. (2010). Die Bedeutung von Verarbeitungstiefe im Musikunterricht - Dimensionen von Unterrichtsqualität in einer Musikstunde. In C. Wallbaum (Hrsg.), Perspektiven der Musikdidaktik. Drei Schulstunden im Licht der Theorie (S. 63-82). Hildesheim: Olms.

Niessen, A. (2011). „Kein Wert an sich“. Wie Schülerinnen und Schüler das Klassenmusizieren erleben. Diskussion Musikpädagogik Sonderheft, Bd. 3 (S. 41-45).

Praetorius, A.-K., \& Charalambous, C. Y. (2018). Classroom observation frameworks for studying instructional quality: looking back and looking forward. ZDM Mathematics Education, 50(3), 535-553.

Praetorius, A.-K., Herrmann, C., Gerlach, E., Zülsdorf-Kersting, M., Heinitz, B., \& Nehring, A. (2020). Unterrichtsqualität in den Fachdidaktiken im deutschsprachigen Raum - zwischen Generik und Fachspezifik. Unterrichtswissenschaft, 48, 409-446.

Puffer, G., \& Hofmann, B. (2016). FALKO-M: Zur Konzeptualisierung des Professionswissens von Musiklehrkräften. In J. Knigge \& A. Niessen (Hrsg.), Musikpädagogik und Erziehungswissenschaft (S. 107-120). Münster: Waxmann.

Puffer, G., \& Hofmann, B. (2017). FALKO-M: Entwicklung und Validierung eines Testinstruments zum domänenspezifischen Professionswissen von Musiklehrkräften. In S. Krauss, A. Lindl, A. Schulcher, 
M. Fricke, A. Göhring, B. Hifmann, P. Kirchhoff \& R. H. Mulder (Hrsg.), FALKO. Fachspezifische Lehrerkompetenzen. Konzeption von Professionswissenstests in den Fächern Deutsch, Englisch, Latein, Physik, Musik, Evangelische Religion und Pädagogik (S. 245-289). Münster: Waxmann.

Rakoczy, K., Klieme, E., \& Pauli, C. (2008). Die Bedeutung der wahrgenommenen Unterstützung motivationsrelevanter Bedürfnisse und des Alltagsbezugs im Mathematikunterricht für die selbstbestimmte Motivation. Zeitschrift für Pädagogische Psychologie, 12(1), 25-35.

Reusser, K. (2008). Empirisch fundierte Didaktik - didaktisch fundierte Unterrichtsforschung. Zeitschrift für Erziehungswissenschaft, Sonderheft, 9, 219-237.

Rolle, C. (1999). Musikalisch-ästhetische Bildung. Über die Bedeutung ästhetischer Erfahrung für musikalische Bildungsprozesse. Kassel: Bosse.

Rolle, C. (2010). Musikdidaktische Reflexionen: Was heißt musikalische Bildung durch Inszenierung ästhetischer Erfahrungsräume? In C. Wallbaum (Hrsg.), Perspektiven der Musikdidaktik. Drei Schulstunden im Licht der Theorien (S. 233-259). Hildesheim: Olms.

Spychiger, M. (2014). Die schwierige Stelle anlächeln. Zur Entwicklung von Fehlerkultur in der Instrumentalpädagogik. Üben \& musizieren, 31(2), 32-34.

Stöger, C. (2021). Musikbezogene Praktiken und die sich darin ausbildenden Kompetenzen sind vielfältig und in ständiger Veränderung begriffen. In V. Krupp (Hrsg.), Wirksamer Musikunterricht (S. 139-148). Hohengehren: Schneider.

Thiel, F., Richter, S. G., \& Ophardt, D. (2012). Steuerung von Übergängen im Unterricht. Eine ExpertenNovizen-Studie zum Klassenmanagement. Zeitschrift für Erziehungswissenschaft, 15(4), 727-752.

Venus, D. (1969). Unterweisung im Musikhören. Wuppertal: Henn.

Wagner, W., Helmke, A., \& Rösner, E. (2009). Deutsch Englisch Schülerleistungen international. Dokumentation der Erhebungsinstrumente für Schülerinnen und Schüler, Eltern und Lehrkräfte. Frankfurt am Main: DIPF.

Wallbaum, C. (2005). Klassenmusizieren als einzige musikalische Praxis im Zentrum von Musikunterricht? In H.-U. Schäfer-Lembeck (Hrsg.), Klassenmusizieren als Musikunterricht!? Theoretische Dimensionen unterrichtlicher Praxen. Beiträge des Münchner Symposions 2005. (S. 71-94). München: Allitera.

Wallbaum, C. (2010). Wenn Musik nur in erfüllter Praxis erscheint. Ästhetische und kulturelle Kriterien für die Untersuchung und Gestaltung von Musikunterricht. In C. Wallbaum (Hrsg.), Perspektiven der Musikdidaktik. Drei Schulstunden im Licht der Theorien (S. 83-122). Hildesheim: Olms.

Zülsdorf-Kersting, M. (2020). Qualitätsmerkmale von Geschichtsunterricht. Zum Verhältnis generischer und fachspezifischer Merkmale. Unterrichtswissenschaft, 48, 385-407. 